2 Author 1

3 - Dr Chao Yang

4 - China Transportation research Institute at Tongji, Tongji University, Shanghai, China

5 - ORCID number

6 Author 2

- Xinyuan Zhang

- School of Transportation Engineering, Tongji University, Shanghai, China

- ORCID number

Author 3

- Dr Rasa Remenyte-Prescott

- Resilience Engineering Research Group, University of Nottingham, UK

- 0000-0003-2881-4017

Dr Rasa Remenyte-Prescott, Resilience Engineering Research Group, University of Nottingham, University Park, Nottingham, NG7 2RD, UK, r.remenyteprescott@nottingham.ac.uk

\title{
Abstract
}

Model (NTFM) for both urban and motorway road networks. It highlights how road maintenance works can be modelled using a roadwork node in the network, and how the modelling can be used to predict the effects of maintenance works on the level of service of the highway network. In particular, the paper aims to show how the theory of vehicle flow fluctuation can be used within the roadwork node for better representation of reality while modelling road network flow. The NTFM is illustrated using traffic characteristics and road maintenance works on a motorway and trunk highway network in China. It is shown how the proposed method is useful in comparing worksite arrangements and describing the effects on flow rates due to roadworks. 
Keywords: road maintenance, roadwork zone arrangements, network-level traffic flow model, the theory of vehicle flow fluctuation

\section{List of notations}

$\omega \quad$ wave velocity

$v_{1} \quad$ the travel speed of the traffic flow before entering the worksite

$v_{2} \quad$ the travel speed of the traffic flow on the worksite

$v_{3} \quad$ the travel speed of the traffic flow leaving the worksite

$v_{4} \quad$ the travel speed of the traffic flow on the worksite at the end of the maintenance activity

$Q_{1} \quad$ the traffic flow entering the worksite

$Q_{2} \quad$ the traffic flow on the worksite

$Q_{3} \quad$ the traffic flow leaving the worksite

$Q_{4} \quad$ the traffic flow on the worksite at the end of the maintenance activity

$L \quad$ length of the worksite

$T \quad$ maintenance duration of the woksite

$t \quad$ the maintenance duration and the time period that the traffic state recovered to normal condition

a coefficient to describe the time saved by crossover strategy

B velocity interference factor

$R_{T} \quad$ the time ratio of the traffic delays under two roadwork zone arrangements

\section{Introduction}

During the last 30 years, China has developed its highway infrastructure. By the end of 2017, there are 4,773,500 km long highways and 136,500 km long motorways in China (Ministry of Transport, China, 2019a). In recent years highways agencies have turned their attention from construction of new roads to maintenance and rehabilitation of existing ones (Ministry of Transport, China, 2017). Large funds are required to deal with pavement deterioration with 
the aim of maintaining the large-sized China highway network in good condition. It is known that the cost of travel delay to road users usually exceeds the cost of maintenance (Ministry of Transport, China, 2019b). In order to alleviate travel delays occurred on the highway network, especially when maintenance activities are taking place, modelling tools, such as the networklevel traffic flow model (NTFM), can be utilised to achieve a better decision-making process for maintenance arrangements, taking into account both maintenance costs and travel delays (Yang, Remenyte-Prescott et al. 2014, Yang, Remenyte-Prescott et al. 2015). The NTFM can be used to model the traffic flow rates, travel speed, and queue dynamics in the highway network under various traffic conditions, making it a suitable platform for setting maintenance arrangements.

Many macroscopic transportation models have been constructed to evaluate traffic conditions on the highway network. Lighthill and Whitham (1955) and Richards (Richards 1956) established the Lighthill-Whitham—Richards (LwR) model based on first order differential equations to analyse the traffic conditions on a single carriageway with the adoption of comparability of 'traffic flow on long crowded roads' with 'flood movements in long rivers'. Payne (Payne 1971) advanced the macroscopic traffic model to a second-order model in which the dynamic flow phenomena were modelled. Later, cell transmission model (CTM) was proposed by Daganzo (Daganzo 1994) to evaluate the traffic on a road section with a single entrance and exit. CTM has been improved to enhance its applicability (Daganzo 1995, Lo 1999, Lo 2001, Lo, Chang et al. 2001). However, only the road network composed of signalised junctions can be modelled by CTM. Some work has been devoted to investigate the traffic at priority junctions, e.g. gap acceptance theory and queuing theory (Kremser 1962, 
Kremser 1964, Cowan 1975, Brilon, Koenig et al. 1999, Troutbeck and Kako 1999, Troutbeck 2002), and some of these findings can be inefficient when modelling directional flow and identifying traffic behaviours at network level (Robinson, Tian et al. 1999, Tian, Troutbeck et al. 2000, Tracz and Gondek 2000, Ruskin and Wang 2002). In order to make more realistic traffic simulations, Yang et al. (Yang, Remenyte-Prescott et al. 2014, Yang, RemenytePrescott et al. 2015) established NTFM to comprise most of the junction types, such as signalised junctions, priority junctions and motorway onramps and offramps, and to predict the traffic flow rates, queues, and travel delays at network level for both urban and motorway road networks.

The novelty of this paper is that the theory of vehicle flow fluctuation is utilised in the roadwork node model of the NTFM, previously developed by the authors (Yang, Remenyte-Prescott et al. 2014, Yang, Remenyte-Prescott et al. 2015), which is then applied to evaluate traffic conditions on the highway network under various roadwork zone arrangements. This approach gives an opportunity to model road network flows more realistically, and it can be further used to optimise road maintenance, for example, to find the best roadwork zone arrangements, considering the entering traffic flows, time saving coefficient and speed interference on the road section. The roadwork node model and its underlying methodology is described in section 2, and the NTFM application and numerical performance on the local highway network in China under different roadwork zone arrangements is provided in section 3. Final remarks about the modelling technique are given in section 4. 


\section{Overview of Network-level Traffic Flow Model (NTFM)}

101 Inthe NTFM, the network is modelled in terms of links, representing roads, and nodes

102 between them, representing junctions.

103

104

\subsection{Junction models in NTFM}

Traffic flow is calculated through the use of junction sub-models, when separate lanes are modelled on the road links, and the flow capacity and the capacitance on each lane are considered. A set of models are established to simulate the traffic dynamics at junctions, as shown in Table 1. For signalised junctions, the traffic flows from each arm are controlled by traffic lights. As for the priority junctions, the evaluation of traffic is based on right-of-way rules, where the entering traffic flow for each arm of the junction is influenced by the flow capacity and the conflicting traffic flows from competing arms. For one-way junctions, except the on-ramp, entering flow is only limited by the road section flow capacity, while on-ramp is evaluated as a priority junction, since the traffic on the ramp must allow the traffic on the major road to pass before it can join the major road.

Table 1 Junction models in the NTFM

\begin{tabular}{l|lll}
\hline Junction groups & Junction types & \\
\hline Signalised Junctions & Signalised T-junction & Signalised Intersection & Signalised Roundabout \\
Priority Junctions & T-junction & Urban Roundabout & Motorway Roundabout \\
One-way Junctions & On-ramp and Off-ramp & Merge and Diverge & Roadwork node \\
\hline
\end{tabular}

\subsection{Roadwork node model}

A roadwork node model is incorporated into the NTFM model to evaluate maintenance activities. The implementation of maintenance work on a road link can be represented by limiting the exiting traffic flow capacity of this link. However, the length and location of worksite, that are used to define the geometry of worksite, also need to be considered, as 
does the link capacitance which changes during maintenance. Therefore, in the NTFM a special roadwork node is employed to represent the part of road link under maintenance, which specifies the location of worksite, the length of the worksite, and the number of lanes in service. A typical roadwork node is shown in Figure 1.

\subsubsection{The worksite arrangements of the dual 2 lane carriageway under maintenance}

Note that a single carriageway is a road with no central reservation to separate opposing flows, whereas a dual carriageway has central reservation. As for a dual 2 lane carriageway, a one-lane closure or a two-lane closure can be used to implement the maintenance actions, which are depicted in Figure $2 a$ ) and $2 b$ ) respectively. For a one-lane closure, only one of the service lanes in one direction is closed owing to maintenance activity, in this case maintenance has no impact on the traffic flow in the opposite direction. While for a two-lane closure, both of the service lanes in one direction are closed, and the road at the worksite becomes effectively a single carriageway.

\subsubsection{The theory of vehicle flow fluctuation applied in the roadwork node model}

The interface between two different densities of traffic flow is called the traffic flow wave, and the velocity of traffic flow wave moving along the road is called the wave velocity. The theory of vehicle flow fluctuation can directly and accurately represent the delay time and queue length generated in vehicle operation. In this paper, we define:

$\omega=v_{1} v_{2}\left(Q_{2}-Q_{1}\right) /\left(Q_{2} v_{1}-Q_{1} v_{2}\right)$

1.

where

$\omega \quad$ wave velocity

$v_{1} \quad$ the travel speed of the traffic flow before entering the worksite

$v_{2} \quad$ the travel speed of the traffic flow on the worksite

$Q_{1} \quad$ the traffic flow entering the worksite 
$Q_{2} \quad$ the traffic flow on the worksite

\subsubsection{One-lane closure}

143 One-lane closure is a very simple roadwork arrangement, in which one-lane is maintained

144 before the other. In the case of two lanes in one direction, it is assumed that the vehicle delay

145 caused by the maintenance of the left lane is the same as the maintenance of the right lane,

146 and the total time required for the maintenance is equal.

147 We assume that the length of the worksite is $L$ and the travel delay time due to road

148 maintenance is $T$, the traffic flow and speed of each section are represented by $Q_{i}, v_{i}$, the

149 wave velocity is expressed by $\omega_{i}$, and then a number of cases to calculate the wave velocity

150 are defined:

$151 \omega_{1}=v_{1} v_{2}\left(Q_{2}-Q_{1}\right) /\left(Q_{2} v_{1}-Q_{1} v_{2}\right)$

1522.

$153 \omega_{3}=v_{2} v_{4}\left(Q_{4}-Q_{2}\right) /\left(Q_{4} v_{2}-Q_{2} v_{4}\right)$

1543.

$155 \omega_{4}=v_{3} v_{4}\left(Q_{4}-Q_{3}\right) /\left(Q_{4} v_{3}-Q_{3} v_{4}\right)$

1564.

$157 \omega_{5}=v_{1} v_{4}\left(Q_{4}-Q_{1}\right) /\left(Q_{4} v_{1}-Q_{1} v_{4}\right)$

1585.

$159 \omega_{6}=v_{2} v_{3}\left(Q_{3}-Q_{2}\right) /\left(Q_{3} v_{2}-Q_{2} v_{3}\right)$

1606.

161 where

$\omega_{1} \quad$ Velocity of delay wave caused by maintenance at the beginning of worksite

$\omega_{3} \quad$ Velocity of dissipative wave when maintenance ends at the beginning of worksite

$\omega_{4} \quad$ Velocity of dissipative wave when maintenance ends at the end of worksite

$\omega_{5} \quad$ Velocity of delay wave when $\omega_{1}$ meets $\omega_{3}$ first.

$\omega_{6} \quad$ Velocity of dissipative wave when $\omega_{3}$ meets $\omega_{4}$ first.

162 For example, if road maintenance section is long enough, $\omega_{4}$ cannot reach the position of $\omega_{3}$ 
before it catches up with $\omega_{1}$, so $\omega_{1}$ and $\omega_{3}$ will intersect first. At this point, the upstream cross-sectional flow is $Q_{4}$ and the downstream cross-sectional flow is $Q_{1}, \omega_{5}$ is formed, as shown in Figure 3(a) where the horizontal axis represents time, while the vertical axis represents distance. If road maintenance section is short, $\omega_{4}$ will pass through the road maintenance section quickly and reach the position of $\omega_{3}$ before $\omega_{3}$ meet $\omega_{1}$. At this moment, upstream cross-sectional flow is $Q_{3}$ and the downstream cross-sectional flow is $Q_{2}$, having a new wave velocity $\omega_{6}$, as shown in Figure $3(b)$. At point $A$, the delay caused by road maintenance will completely dissipate, so the abscissa of point $A$ is the total delay time caused by road maintenance to traffic flow.

By geometric relations:

a. if $L>2 T\left|\omega_{1}\right|\left(\omega_{4}-\omega_{3}\right) /\left(\omega_{3}-\omega_{1}\right)$, then traffic delay time due to road maintenance, $t a$, is $t a=\left(L\left(\omega_{3}-\omega_{1}\right)+2 T\left(\omega_{1} \omega_{4}-\omega_{1} \omega_{3}-\omega_{3} \omega_{4}+\omega_{3} \omega_{5}\right)\right) /\left(\omega_{1} \omega_{4}-\omega_{1} \omega_{5}-\omega_{3} \omega_{4}+\omega_{3} \omega_{5}\right)$ 7.

b. if $L<2 T\left|\omega_{1}\right|\left(\omega_{4}-\omega_{3}\right) /\left(\omega_{3}-\omega_{1}\right)$, then Traffic delay time due to road maintenance, $t b$, is $t b=\left(L\left(\omega_{3}-\omega_{5}\right)+2 T\left(\omega_{3}^{2}-\omega_{3} \omega_{4}-\omega_{3} \omega_{5}+\omega_{4} \omega_{5}\right)\right) /\left(\omega_{1} \omega_{3}-\omega_{1} \omega_{4}-\omega_{3} \omega_{5}+\omega_{4} \omega_{5}\right)$ 8.

\subsubsection{Two-lane closure and crossover}

The crossover strategy will maintain all lanes in one direction at a time, and the traffic flow will travel across the central divider. This will reduce the maintenance duration but will cause additional delays in both directions. 
Two more parameters are introduced to simulate the real traffic characteristics. It is known that the total maintenance time for the worksite under the crossover maintenance scenario is coefficient for time saved by the crossover strategy) is introduced to indicate the time benefits of the crossover strategy. During the crossover strategy, the traffic needs to cross the central divider, but the lack of the divider in the opposite direction leads to the mutual influence of vehicle in both directions, which result in the speed reduction, denoted by $\beta$ (velocity interference factor). The traffic condition under the crossover strategy is shown in Figure 4. Figure 4(b) represents the side of the road with the worksite; Figure 4(a) represents the opposite side of the road. As above, using the abscissa of point A as the total delay time due to maintenance work gives the following expressions:

\section{c. In the maintenance direction:} $t c=2 T \alpha \omega_{6} /\left(\omega_{6}-\omega_{1}\right)$

d. In the opposite direction with $L>2 T\left|\omega_{1}\right|\left(\omega_{4}-\omega_{3}\right) /\left(\omega_{3}-\omega_{1}\right)$, $t d=\left(L v_{2} \beta\left(\omega_{3}-\omega_{1}\right)+L \omega_{1}\left(\omega_{4}-\omega_{3}\right)+L \omega_{3}\left(\omega_{5}-\omega_{4}\right)\right.$

$$
\left.+2 T v_{2} \alpha \beta\left(\omega_{1} \omega_{4}-\omega_{1} \omega_{3}-\omega_{3} \omega_{4}+\omega_{3} \omega_{5}\right)\right)
$$


$t e=\left(L v_{2} \beta\left(\omega_{3}-\omega_{6}\right)+L \omega_{6}\left(\omega_{4}-\omega_{3}\right)+2 T v_{2} \alpha \beta\left(\omega_{6} \omega_{4}-\omega_{3} \omega_{6}\right)\right)$

$$
/ v_{2} \beta\left(\omega_{1} \omega_{3}-\omega_{1} \omega_{4}-\omega_{3} \omega_{5}+\omega_{4} \omega_{5}\right)
$$

11.

\subsubsection{The comparison of traffic delays under two roadwork zone arrangements}

212 In conclusion, if $L>2 T\left|\omega_{1}\right|\left(\omega_{4}-\omega_{3}\right) /\left(\omega_{3}-\omega_{1}\right)$, the total traffic delay for the two opposite

213 directions under the two-lance closure and crossover strategy is denoted as

$214 t_{\text {cross }}=t c+t d$,

21512.

216 and the time ratio of the traffic delays under two roadwork arrangements is

If $L<2 T\left|\omega_{1}\right|\left(\omega_{4}-\omega_{3}\right) /\left(\omega_{3}-\omega_{1}\right)$, the total traffic delay of the two opposite directions under the two-lance closure and crossover strategy is calculated as

\section{Model application}

228 To illustrate the performance of the NTFM, using the theory of vehicle flow fluctuation, a case 
in China is presented, as shown in Figure 5. The highway network includes both urban road links and motorway links, which is composed of 7 road sections and 6 junctions, i.e. 4 motorway links, 3 urban road links, 2 on-ramps, 2 off-ramps, and 2 signalised intersections. Roadworks are introduced to demonstrate the effects of the two worksite arrangements, and the sensitivity of the two parameters a (the coefficient of time saved by the crossover strategy) and $\beta$ (the velocity interference factor) is analysed concurrently.

Three points (,$B$ and $C$ ) are selected as the maintenance worksites on the network, and the data needed for the model is obtained through traffic survey. In order to estimate the traffic delay caused by the maintenance, some appropriate assumptions are made:

a) if maintenance leads to congestion, the traffic flow in the maintenance section will reach the saturated flow of the single lane, and the driving speed will be reduced to the prescriptive speed limit;

b) the traffic flow going through the maintenance section should be larger than the flow entering the section, otherwise the delay will not dissipate, so it is assumed that the leaving flow is slightly greater than the flow before entering the worksite. Note that these assumptions are based on the relationship between velocity and density from the Greenshields model (Greenshields et al., 1935);

c) when the vehicle leaves the maintenance section, its speed will increase due to a better flow as fewer vehicles will be present. In addition, the speed value is assumed to be between the speed before entering the maintenance section and the speed of driving through the maintenance section; d) when roadworks are completed, due to the availability of the lane(s), it will be some time 
before the speed will increase, therefore, the traffic flow will be close to saturation.

e) the traffic flow entering the worksite is constant,

254 Road network maintenance data and the values of model parameters are given in Table 2

255 and 3 respectively.

Table 2 Road network maintenance data

\begin{tabular}{cccccc}
\hline Survey Point & Direction & $Q_{1}(p c u / h)$ & $v_{1}(k m / h)$ & $\begin{array}{l}\text { L(Brinkmann, } \\
\text { Ulmer et al. })\end{array}$ & T(h) \\
\hline \multirow{2}{*}{ A } & $1^{*}$ & 2500 & 80 & 1 & 4 \\
& 2 & 2500 & 80 & & 4 \\
B & $1^{*}$ & 2400 & 80 & 1 & 4 \\
& 2 & 2000 & 90 & & 4 \\
\hline
\end{tabular}

* road section under maintenance

Table 3 The setting of parameters

\begin{tabular}{ccccc}
\hline \multirow{2}{*}{$\boldsymbol{Q}_{\boldsymbol{i}}(\boldsymbol{p} \boldsymbol{c u} / \boldsymbol{h})$} & $Q_{1}$ & $Q_{2}$ & $Q_{3}$ & $Q_{4}$ \\
\cline { 2 - 5 } & $Q_{1}$ & $L q=1800$ & $1.1 Q_{1}$ & 3000 \\
\hline \multirow{2}{*}{$\boldsymbol{v}_{\boldsymbol{i}}(\boldsymbol{k m} / \boldsymbol{h})$} & $v_{1}$ & $v_{2}$ & $v_{3}$ & $v_{4}$ \\
\cline { 2 - 5 } & $v_{1}$ & $10,15,20$ & $\left(v_{1}+v_{2}\right) / 2$ & $1.5 v_{2}$ \\
\hline
\end{tabular}

$* L q$ is the flow capacity for each lane

It is assumed that the length of the maintenance section at the three worksites, A, B and C,

is $1 \mathrm{~km}$ long and the time required to conduct the maintenance is 4 hours. In Table 2, the are given. In Table 3, the values of the traffic flow and travel speed are presented at three other locations (in addition to entering the worksite): on the worksite ( $Q_{2}$ and $v_{2}$ ), leaving the worksite $\left(Q_{3}\right.$ and $\left.v_{3}\right)$ and on the worksite after the maintenance activity is completed $\left(Q_{4}\right.$ and $\left.V_{4}\right)$.

\subsection{Worksite A}

In worksite $A$, the maintenance direction and the opposite direction experienced the same 
steady flow before entering the worksite, travel delay time and time ratio of the delays under two different worksite strategies can be calculated, expressed by using variables $\alpha, \beta, v_{2}$. The abscissa in Figure 6 represents the variation of inflows to the worksite, the ordinate in the graph on the left represents the travel delays and the ordinate in the graph on the right represents the time ratio under two worksite arrangements. It can be seen in Figure 6 that the higher the traffic flow entering the maintenance section, the higher the delay; the lower the speed limit in the maintenance section, the higher the delay. Furthermore, in the crossover roadwork arrangement, the speed reduction due to a lack of central divider will also increase the delay. As shown in the graphs on the right of Figure 6, the time ratio, $\alpha$, of the traffic delays under two worksite arrangements formed horizontal lines when $\beta$ equals to one, this is due to the fact that $v_{2}$ values are the same under the two different arrangements. As the benchmark determined by $\alpha$, variable $\beta$ will cause a different growth trend in the time ratio. Smaller values of $\beta$ mean that more serious interference of velocity during the maintenance will lead to longer delays, especially in the case of high values of entering flow. Even without considering the speed reduction, the total delay (Cross A and Cross B) in the crossover roadwork arrangement is still slightly larger than in the one-lane closure roadwork arrangement, but the delay of single direction are both less than one-lane closure. Therefore, in worksite $\mathrm{A}$, one-lane closure will cause a smaller travel delay than the crossover strategy.

\subsection{Worksites B and C}

In worksites $\mathrm{B}$ and $\mathrm{C}$, flow rates in the maintenance direction and in the opposite direction are different. For example, the flow in the maintenance direction of worksite B is larger than the flow in the opposite direction, which is in contrary to worksite C. As above, travel delay time 
and time ratio of the delays under two different roadwork arrangements can be calculated.

In Figure 7 the abscissa describes $D i r_{1} / D i r_{2}$, which is used to represent the ratio between the entering flow in maintenance direction, $D i r_{1}$, and in the opposite direction, $D i r_{2}$. It can be seen in Figure 7 that the travel delay on the opposite side will increase due to the increase of the ratio of entering flow in maintenance direction and the opposite direction, but the maintenance side delay is only affected by $\alpha, \beta$ in defining $v_{2}$. Again, as shown in the graphs on the right of Figure 7, $\alpha$ forms a cluster of baselines and $\beta$ decides the growth trend of the time ratio. If the traffic flow on both sides is asymmetrical, the crossover option might have its advantages compared to the one-lane closure option. If the opposing traffic flow is small, taking over the opposite lanes will not cause too much delay, therefore, the total delay will be relatively low. However, the crossover maintenance worksite arrangement becomes desirable only when the opposite flow is lower than the flow in the maintenance direction, which would require at least twice as much materials and manpower. Therefore, during the decisionmaking for worksite $\mathrm{B}$, the values of $\alpha, \beta$, need to be considered, while for worksite $\mathrm{C}$, the one-lane closure strategy should be adopted to avoid unnecessary congestion.

\section{Discussion}

The roadwork node model within the NTFM is used to forecast the traffic movement at the worksite, and the application of the method is demonstrated on the Shanxi-Neimenggu highway network in China, considering commonly observed worksite zone arrangements. In the analysis of the results, it can be seen the suitability of the worksite zone arrangements is affected by the traffic flow on the network. Specifically, the crossover strategy is more suitable for a situation that the traffic flow in the opposite direction to the worksite flow is low, because 
the lane occupancy will not cause any long delays and the total delay in both directions will be smaller than the one resulted in by the one-lane closure strategy. However, this result is only true under certain conditions. For example, it is required that in the crossover strategy the time needed to carry out maintenance of both lanes in one direction is similar to the time needed to carry out maintenance of one lane in the one-lane closure strategy. This scenario requires not only twice as many road workers, but also that the lanes are independent of each other, which means that it can take twice as many workers to shorten the maintenance time by $50 \%$. In addition, such an analysis is based on the insufficient capacity of a single lane. Finally, the difference in cost between the two worksite arrangements is not considered in this paper, only the delay time is taken into account, and most values of the parameters are assumed for illustration purposes. Therefore, this analysis method is useful while exploring worksite arrangements for parts of road network that are sensitive to traffic congestion, and more detailed traffic flow and worksite arrangement information would be necessary to derive the values of model parameters.

\section{Conclusions}

This paper is based on the network traffic flow model (NTFM) which is used to calculate traffic flows, especially when additional delays on the network are caused by road maintenance.

332 The main novel features of this study are listed below:

333 - The theory of vehicle flow fluctuation is incorporated into the NTFM, more specifically, to

334 model roadworks, and the model is used to calculate the delay time and queue length, generated by the worksite. 
- The traffic delays on the road section under the one-lane closure and the two-lane closure strategies are investigated, considering three worksites with different features.

- The sensitivity analysis of the coefficient of time saved by the crossover strategy, $a$, and the velocity interference factor, $\beta$, are presented.

- When the traffic flows on both sides are asymmetrical and the flow rate from the worksite zone direction is relatively high, the two-lane closure and the crossover arrangement on the worksite is preferable; otherwise, the delay in the two-lane closure and the crossover arrangement is larger than in the one-lane arrangement.

In addition, a case study has been carried out in order to illustrate how traffic conditions on the Shanxi-Neimenggu highway network in China can be investigated. Traffic flows and queues throughout the worksite on the network were calculated under various roadwork zone arrangements. It is shown how the proposed method is capable of evaluating the traffic condition of a road network and to demonstrate disturbances due to maintenance activities at a network level. Further research could be carried out in the area of optimisation of maintenance arrangements when the inflows to the maintenance area change over time. Further detailed cases of roadwork zone arrangements could also be modelled, such as speed restrictions in the one-lane closure case and more detailed modelling of traffic flow at the end of the roadwork in the two-lane closure case.

\section{References}

Brilon, W., R. Koenig and R. J. Troutbeck (1999). "Useful estimation procedures for critical gaps." Transportation Research Part A: Policy and Practice $33(3-4)$ : 161186.

Brinkmann, J., M. W. Ulmer and D. C. Mattfeld (2015). "Short-term Strategies for Stochastic Inventory Routing in Bike Sharing Systems." Transportation Research Procedia 10: 364-373. 
Cowan, R. J. (1975). "Useful headway models." Transportation Research 9(6) : 371-375. Daganzo, C. F. (1994). "The Cell Transmission Model - a Dynamic Representation of Highway Traffic Consistent with the Hydrodynamic Theory." Transportation Research Part B-Methodological 28(4): 269-287.

Daganzo, C. F. (1995). "The Cell Transmission Model .2. Network Traffic." Transportation Research Part B-Methodological 29(2) : 79-93.

Greenshields, B. d., J. r. Bibbins, W. s. Channing and H. h. Miller (1935). "A study of traffic capacity." Highway Research Board proceedings 1935:

Kremser, H. (1962). "Ein Zusammengesetztes Wartezeitproblem Bei Poissonschen Verkehrsströmen (A Complex Problem of Delay with Poisson-Distributed Traffic Flows)." Osterreichisches Ingenieur-Archiv 16.

Kremser, H. (1964). "Wartezeiten Und Warteschlangen Bei Einfadelung Eines Poissonprozesses in Einen Anderen Solchen Prozess (Delays and Queues with One Poisson Process Merging into Another 0ne)." Österreichisches Ingenieur-Archiv 18.

Lo, H. K. (1999). "A novel traffic signal control formulation." Transportation Research Part A: Policy and Practice 33(6): 433-448.

Lo, H. K. (2001). "A Cel1-Based Traffic Control Formulation: Strategies and Benefits of Dynamic Timing Plans." TRANSPORTATION SCIENCE 35(2) : 148-164.

Lo, H. K., E. Chang and Y. C. Chan (2001). "Dynamic network traffic control." Transportation Research Part A: Policy and Practice 35(8) : 721-744.

Ministry of Transport, China (2017). The 13th five-year plan for the development of the modern comprehensive transport system, 3rd Feb, 2017, Beijing, China.

Ministry of Transport, China (2019a). 2018 transport work conference, 11th Jan, 2019, Beijing, China.

Ministry of Transport, China (2019b). 2018 transport statistics, 12th Apri1, 2019, Beijing, China.

Payne, H. J. (1971). "Models of freeway traffic and control. ." Simulation council proceeding 1: ch6: $51-61$.

Richards, P. I. (1956). "Shock Waves on the Highway." Operations Research 4(1): 4251.

Robinson, B. W., Z. Tian, W. Kittelson, M. Vandehey, M. Kyte, W. Brilon, N. Wu and R. Troutbeck (1999). "Extensions of theoretical capacity models to account for special conditions." Transportation Research Part A: Policy and Practice $33(3-4)$ : $217-236$.

Ruskin, H. J. and R. Wang (2002). Modeling Traffic Flow at an Urban Unsignalized Intersection. Proceedings of the International Conference on Computational SciencePart I, Springer-Verlag: 381-390.

Tian, Z. Z., R. Troutbeck, M. Kyte, W. Brilon, M. Vandehey, W. Kittelson and B. W. Robinson (2000). "A further investigation on critical gap and follow-up time " TRansportation Research Circular E-C108: 4th International Symposium on Highway Capacity 397-408.

Tracz, M. and S. Gondek (2000). "Use of simulation to analysis of impedance impact at unsignalized intersections." Transportation Research Circular (E-C018) : 471-484.

Troutbeck, R. (2002). "The performance of uncontrolled merges using a limited 
priority process." Transportation and Traffic Theory in the 21st Century: 463-482. Troutbeck, R. J. and S. Kako (1999). "Limited priority merge at unsignalized intersections." Transportation Research Part A: Policy and Practice 33(3-4): 291304.

Yang, C., R. Remenyte-Prescott and J. Andrews (2014). "A network traffic flow model for motorway and urban highways." J Oper Res Soc 65(8) : 1278-1291.

Yang, C., R. Remenyte-Prescott and J. Andrews (2015) "Application of network traffic flow model to road maintenance." Proceedings of the ICE - Transport 168, 256-266.

Figure 1. A road section under repair

Figure 2. Dual 2 carriageway worksite arrangements (a) One-lane closure, (b) Two-lane closure and crossover Figure 3. One-lane closure space-time (a) Long worksite, (b) Short worksite Figure 4. Two-lane closure and crossover space-time (a) Maintenance direction, (b) The opposite direction with long section, (c) The opposite direction with short section Figure 5. Shanxi and Neimenggu road network Figure 6 Different maintenance decisions with balance direction (a) $v_{2}=10 \mathrm{~km} / \mathrm{h}$, (b) $v_{2}=$ $15 \mathrm{~km} / \mathrm{h},(\mathrm{c}) v_{2}=20 \mathrm{~km} / \mathrm{h}$

Figure 7 Different maintenance decisions with asymmetrical flow (a) $v_{2}=10 \mathrm{~km} / \mathrm{h}, \quad$ (b) $v_{2}=$ $15 \mathrm{~km} / \mathrm{h},(\mathrm{c}) v_{2}=20 \mathrm{~km} / \mathrm{h}$ 